Kompass

Autoimmun

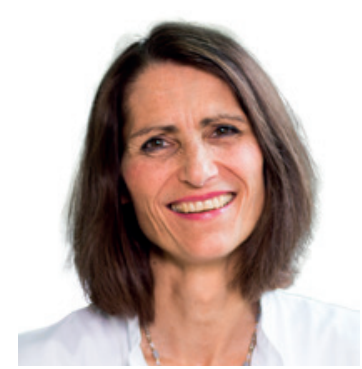

\title{
Chemokine in der Orchestrierung des Typ-1-Diabetes: Pathophysiologie und neuer Therapieansatz?
}

\author{
Sabine Adler
}

Fachbereich Gastroenterologie/Hepatologie, Endokrinologie/Diabetologie, Rheumatologie, Helios Klinikum Erfurt, Erfurt, Deutschland

Abstract aus Christen U, Kimmel R: Chemokines as drivers of the autoimmune destruction in type 1 diabetes: opportunity for therapeutic intervention in consideration of an optimal treatment schedule. Front Endocrinol (Lausanne) 2020;11:591083.

\section{Keywords}

CD3 · CXCL10 · CXCR3 · Combination therapy · Insulitis · Migration

\begin{abstract}
Type 1 diabetes (T1D) is mainly precipitated by the destruction of insulin-producing $\beta$-cells in the pancreatic islets of Langerhans by autoaggressive T cells. The etiology of the disease is still not clear, but besides genetic predisposition the exposure to environmental triggers seems to play a major role. Virus infection of islets has been demonstrated in biopsies of T1D patients, but there is still no firm proof that such an infection indeed results in islet-specific autoimmunity. However, virus infection results in a local inflammation with expression of inflammatory factors, such as cytokines and chemokines that attract and activate immune cells, including potential autoreactive T cells. Many chemokines have been found to be elevated in the serum and expressed by islet cells of T1D patients. In mouse models, it has been demonstrated that $\beta$-cells express chemokines involved in the initial recruitment of immune cells to the islets. The bulk load of chemokines is however released by the infiltrating immune cells that also express multiple chemokine receptors. The result is a mutual attraction of antigen-pre-
\end{abstract}

senting cells and effector immune cells in the local islet microenvironment. Although there is a considerable redundancy within the chemokine ligand-receptor network, a few chemokines, such as CXCL10, seem to play a key role in the T1D pathogenesis. Studies with neutralizing antibodies and investigations in chemokinedeficient mice demonstrated that interfering with certain chemokine ligand-receptor axes might also ameliorate human T1D. However, one important aspect of such a treatment is the time of administration. Blockade of the recruitment of immune cells to the site of autoimmune destruction might not be effective when the disease process is already ongoing. By that time, autoaggressive cells have already arrived in the islet microenvironment and a blockade of migration might even hold them in place leading to accelerated destruction. Thus, an anti-chemokine therapy makes most sense in situations where the cells have not yet migrated to the islets. Such situations include treatment of patients at risk already carrying islet-antigen autoantibodies but are not yet diabetic, islet transplantation recipients, and patients that have undergone a $\mathrm{T}$ cell reset as occurring after anti-CD3 antibody treatment. 


\section{Transfer in die Praxis}

\section{Einführung/Hintergrund}

Diabetes galt lange Zeit als genetisch determiniertes Schicksal. Mittlerweile ist klar, dass es sich im Wesentlichen um eine autoimmune Störung handelt und autoreaktive CD8+ T-Zellen zur Destruktion der $\beta$-Zellen des Pankreas führen. Weniger klar ist jedoch, wie die Aktivierung der T-Zellen ausgelöst worden ist und ob man diese dann auch therapeutisch «umlenken» oder blockieren kann. Neben der Mutmaßung einer genetisch unterliegenden Veranlagung wird auch hier - wie bei vielen autoimmunen Problemen ein sogenannter «second hit» vermutet: Enteroviren werden hier als ein möglicher «zweiter Auslöser» der Immunkaskade, analog zu einem Dominospiel, diskutiert. Letztlich ist diese Theorie jedoch nie klar zu beweisen und natürlich im humanen Organismus experimentell nicht nachstellbar.

Dennoch kann eine solche (oder andere) Viruserkrankung dazu führen, dass Virusanteile als körperähnlich wahrgenommen werden und so die Immuntoleranz zugunsten des Virus durchbrochen wird. Dieses als «molecular mimicry» beschriebene Phänomen kann somit eine negative Immunkaskade auslösen.

\section{Chemokine sind ein treibender Faktor in der Entwicklung des Typ-1-Diabetes}

Zelluläre Infiltrationen in akut oder chronisch entzündlich verändertes Gewebe - wie auch bei Autoimmunprozessen - werden über Chemokine vermittelt. Diese Vermittler werden überwiegend vom ortsständigen Endothel und einwandernden Immunzellen freigesetzt. Die daraus resultierenden Krankheitsentitäten zeigen hierbei häufig ein krankheitstypisches, charakteristisches Muster dieser Zytokine oder deren Zytokinkaskaden. Im Fall eines Diabetes führt dies zur «lnsulitis», der Inflammation und letztlich zur Destruktion der Langerhans-Zellen.

Untersuchungen dieser Immunkaskaden sind bedeutend, wenn neue Therapien gezielt gegen eine solche wirken sollen. Problematisch ist es jedoch, menschliches Pankreasgewebe hierfür zu gewinnen, sodass die meisten Studien an Mausmodellen oder durch Messungen im menschlichen Serum vorgenommen werden. Darüber hinaus sind Abläufe einer destruierenden Insulitis bei Mäusen sowohl zeitlich als auch vom Ausmaß der Destruktion her nicht klar mit menschlichen Abläufen gleichzusetzen. Und letztlich sind ja auch bei den Betroffenen die Abläufe unterschiedlich lang und schwer. Zahlreiche Arbeiten an menschlichem und tierischem Gewebe wie auch an menschlichen Seren haben dennoch die CXCL10/CXCR3-Achse als häufigste Expression - sozusagen als kleinsten gemeinsamen Nenner - dargestellt.

\section{Kann die Chemokinblockade therapeutisch hilfreich sein?}

Therapeutisch konnte in Mausmodellen gezeigt werden, dass die Neutralisation dieser Chemokine zu einer Reduktion der Ausprägung des Typ-1-Diabetes führen kann. Der Vorteil hierbei ist natür- lich, dass in einer Laborsituation sowohl der Zeitpunkt des (zum Teil induzierten) Krankheitsbeginns als auch die Messung von eventuell vorhandenen Autoantikörpern definiert werden kann. Somit kann hier früh in ein noch nicht voll etabliertes Krankheitsgeschehen eingegriffen werden. Das ist beim Menschen leider nicht klar möglich, sodass hier noch ein frühzeitiger therapeutischer Einsatz der Neutralisation postuliert werden muss.

Da wie erwähnt verschiedene Signalwege an der Entzündungskaskade mitwirken, sind experimentelle Untersuchungen zur sequenziellen Immunblockade unterwegs: Zunächst wird eine T-Zell-Depletion über Anti-CD3-Antikörper angestrebt, in einem weiteren Schritt erfolgt dann die Blockade der CXCL10/CXCR3-Achse. In den entsprechenden Mausmodellen konnte hierdurch bei geringen Nebenwirkungen eine dauerhafte Remission bei den therapierten Mäusen erzielt werden.

\section{Kommentar und Fazit für die Praxis}

Die autoimmune Genese des Typ-1-Diabetes steht klar im Fokus. Als möglicher Hintergrund wird hier die Aktivierung der Chemokinachse aus CXCL10/CXCR3 beschrieben. Auch wenn diese Tatsache zunächst weit entfernt vom klinischen Alltag erscheint, werden sich daraus wahrscheinlich Implikationen für zukünftige Therapien ergeben. Die therapeutische Blockade einzelner Signalwege und einzelner Zytokine ist bei einigen Autoimmunerkrankungen bereits etabliert und stellt eine «elegante», da selektive Alternative zur unselektiven Blockade ganzer Zelllinien mit ansonsten daraus resultierenden Langzeitrisiken der Immunsuppression dar.

Problematisch in der Anwendung bei Typ-1-Diabetes wird jedoch sein, den optimalen Zeitpunkt einer solchen Therapiestrategie finden zu können. Ein frühzeitiger Einsatz in der Phase des «lmmunphänomens» Diabetes vor dem Auftreten einer manifesten Erkrankung wäre wünschenswert, ist jedoch im Alltag bislang schwer abzuschätzen.

Es bleibt also zu hoffen, dass einerseits diese Therapieoption überhaupt weiterentwickelt und anwendbar sein wird und andererseits Biomarker zur Verfügung stehen, die einen optimalen Einsatzzeitpunkt ermöglichen.

Diese Übersichtsarbeit schafft Hoffnung auf eine individualisierte oder «targeted» Therapie des Diabetes Typ 1 und lässt weitere Erkenntnisse zu den zugrunde liegenden Pathomechanismen autoimmuner Erkrankungen erwarten.

\section{Disclosure Statement}

Bezüglich des aktuellen Wissenstransfers bestehen keine Interessenkonflikte.

Korrespondenz an:

PD Dr. Sabine Adler, Sabine.Adler@helios-gesundheit.de 